Acta Crystallographica Section F

Structural Biology

and Crystallization

Communications

ISSN 1744-3091

\section{Kamel El Omari, ${ }^{a}$ Catherine Porcher $^{b}$ and Erika J. Mancini ${ }^{a *}$}

a Division of Structural Biology, The Wellcome Trust Centre for Human Genetics, University of Oxford, Roosevelt Drive, Oxford OX3 7BN, England, and ${ }^{\mathbf{b}} \mathrm{MRC}$ Molecular Haematology Unit, Weatherall Institute of Molecular Medicine, John Radcliffe Hospital, University of Oxford, Oxford OX3 9DS, England

Correspondence e-mail: erika@strubi.ox.ac.uk

Received 5 July 2010

Accepted 16 August 2010

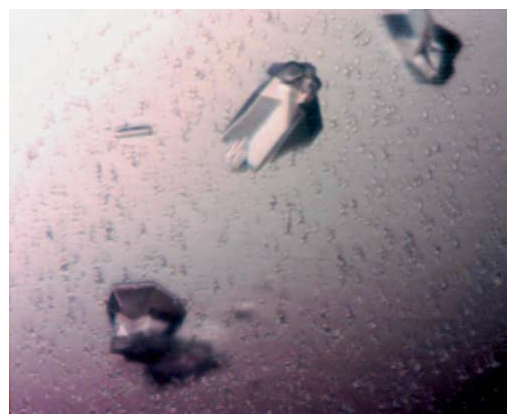

C 2010 International Union of Crystallography All rights reserved

\section{Purification, crystallization and preliminary X-ray analysis of a fusion of the LIM domains of LMO2 and the LID domain of Ldb1}

LMO2 (LIM domain only 2), also known as rhombotin-2, is a transcriptional regulator that is essential for normal haematopoietic development. In malignant haematopoiesis, its ectopic expression in T cells is involved in the pathogenesis of leukaemia. LMO2 contains four zinc-finger domains and binds to the ubiquitous nuclear adaptor protein Ldb1 via the LIM-interaction domain (LID). Together, they act as scaffolding proteins and bridge important haematopoietic transcription factors such as SCL/Tal1, E2A and GATA-1. Solving the structure of the LMO2:Ldb1-LID complex would therefore be a first step towards understanding how haematopoietic specific protein complexes form and would also provide an attractive target for drug development in anticancer therapy, especially for T-cell leukaemia. Here, the expression, purification, crystallization and data collection of a fusion protein consisting of the two LIM domains of LMO2 linked to the LID domain of Ldb1 via a flexible linker is reported. The crystals belonged to space group $C 2$, with unit-cell parameters $a=179.9, b=51.5$, $c=114.7 \AA, \beta=90.1^{\circ}$, and contained five molecules in the asymmetric unit. Multiple-wavelength anomalous dispersion (MAD) data have been collected at the zinc X-ray absorption edge to a resolution of $2.8 \AA$ and the data were used to solve the structure of the LMO2:Ldb1-LID complex. Refinement and analysis of the electron-density map is in progress.

\section{Introduction}

LMO2 (LIM domain only 2) is a member of the LIM-only (LMO) family of LIM domain-containing transcriptional cofactors. LIM domains are 55-residue cysteine-rich structural units composed of two zinc fingers linked by a two amino-acid residue hydrophobic linker. LIM domain-containing proteins are believed to play crucial roles in many essential cellular processes such as cell growth, trafficking, cytoskeletal organization, differentiation and apoptosis (Zheng \& Zhao, 2007; Bach, 2000) by mediating protein-protein interactions through their zinc-finger domains. Specifically, the presence of a tandem of LIM domains in the LMO proteins confers on them the potential to engage in multiple protein-protein interactions.

LMO2 is a 158-amino-acid nuclear protein composed of two LIM domains and a small $\mathrm{N}$-terminal transactivation domain. LMO2 plays a central role in haematopoietic stem-cell development, erythropoiesis and angiogenesis (Warren et al., 1994; Yamada et al., 2000; Yamada et al., 1998). Upon chromosomal translocations or biallelic transcriptional activation, its ectopic expression is involved in the pathogenesis of T-cell acute lymphoblastic leukaemia (T-ALL; Boehm et al., 1991; Ferrando et al., 2004). In normal haematopoiesis, LMO2 interacts with the ubiquitously expressed protein Ldb1 [also known as CLIM (LIM homeobox protein cofactor) or NLI (nuclear LIM-domain interactor)]. Ldb1 comprises a 39-amino-acid C-terminal LIM-interaction domain (LID) that mediates interaction with all LMO proteins and LIM homeodomains (Jurata \& Gill, 1997; Kadrmas \& Beckerle, 2004) and an N-terminal dimerization domain (Jurata \& Gill, 1997) that allows the formation of higher order protein complexes. Indeed, the LMO2:Ldb1 complex acts as a scaffolding protein and participates in the assembly of a DNA-binding multiprotein complex that includes transcriptional regulators such as SCL, E2A and GATA-1 (Lecuyer et al., 2007; Schlaeger et al., 2004; Wadman et al., 1997). When abnormally expressed, similar protein 
complexes are believed to be involved in tumorigenesis in T-ALL (Grutz et al., 1998; Herblot et al., 2000; Ono et al., 1998). Interestingly, a role for LMO2 in B-cell lymphomas (Natkunam et al., 2007) and prostate cancer (Ma et al., 2007) has also been reported. Consequently, LMO2 has become a very attractive anticancer drug target. Efforts are currently focused on designing peptides and/or intrabodies that are able to disrupt transcriptional complexes containing LMO2 (Nam et al., 2008; Appert et al., 2009).

NMR structures of the N-terminal LIM domains of LMO4 and LMO2 in complex with the Ldb1 LID domain (Ldb1-LID; PDB codes $1 \mathrm{j} 2 \mathrm{o}$ and $1 \mathrm{~m} 3 \mathrm{v}$, respectively; Deane et al., 2003) provided the first examples of LIM:Ldb1-LID complexes and highlighted the residues responsible for the interactions of the N-terminal LIM domains. The structure of both LIM domains of LMO4 fused to Ldb1-LID has also been solved by X-ray crystallography (LMO4:Ldb1-LID; PDB code 1rut; Deane et al., 2004); however, the structure of the two-LIM-domain LMO2 in complex with Ldb1-LID has not been reported to date. Despite their $46 \%$ sequence identity, LMO2 and LMO4 have different functions and binding partners and bind Ldb1 with different affinities (Ryan et al., 2006). In order to obtain insights into the mechanism of action of LMO2 and to detail its binding interface with the Ldb1-LID domain, we set out to solve the structure of the LMO2:Ldb1-LID complex. Here, we report its production, crystallization and preliminary diffraction analysis.

\section{Material and methods}

\subsection{Cloning and purification}

The LMO2:Ldb1-LID construct consists of human LMO2 (residues 26-153) fused to the Ldb1-LID fragment (residues 336-368) via an 11-residue linker (GGSGGHMGSGG; Fig. 1). The sequence coding for the fusion protein was obtained as described previously (Deane et al., 2001). Briefly, the LMO2-linker insert was produced by PCR using primers 1 (5'-CGG GAT CCA TCC CTG CTG ACA TGC GGC GG) and 2 (5'-GCC ACC GGA ACC CAT ATG ACC GCC GCT GCC ACC CCC ATT GAT CTT AGT CCA CTC) and linkerLdb1-LID was produced using primers 3 (5'-GGT GGC AGC GGC GGT CAT ATG GGT TCC GGT GGC GAT GTG ATG GTG GTG GGG GA) and 4 (5'-GGA ATT CTC ACT ATT ACT CGT CGT CAA TGC CGT TGG). The full-length construct was obtained by a third PCR using primers 1 and 4. The insert encoding LMO2:Ldb1LID was cloned into the pETduet vector (Novagen) between BamHI and EcoRI restriction sites.

The plasmid pETduet-LMO2:Ldb1-LID encoding N-terminally $6 \times$ His-tagged protein was transformed into Rosetta (DE3) pLysS. Growth was carried out at $310 \mathrm{~K}$ in Luria broth (LB) supplemented with $50 \mu \mathrm{g} \mathrm{ml}^{-1}$ ampicillin until the absorbance at $600 \mathrm{~nm}$ reached

\begin{tabular}{|l|l|}
\hline 26 & \multicolumn{1}{|r|}{153} \\
\hline LIM1 & LIM2 \\
\hline
\end{tabular}

$\mathrm{LMO} 2$

\begin{tabular}{l|l|l|l}
\hline LIM1 LIM2 & GGSGGHMGSGG & LID & LMO2:LDB1-LID \\
\hline
\end{tabular}

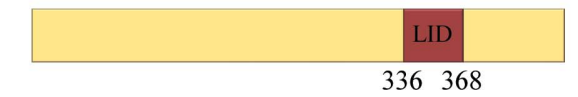

LDB1

Figure 1

Schematic representation of the LMO2:Ldb1-LID fusion protein. In the figure, LMO2 is shown in light blue with its LIM domains in dark blue and Ldb1 is shown in yellow with its LID domain in red.
0.7. Expression of the fusion protein was induced by the addition of $1 \mathrm{~m} M$ isopropyl $\beta$-D-1-thiogalactopyranoside (IPTG) and growth was continued for $16 \mathrm{~h}$ at $303 \mathrm{~K}$. The cells were harvested by centrifugation and resuspended in $50 \mathrm{~m} M$ sodium phosphate $\mathrm{pH}$ 7.4, $500 \mathrm{mM}$ $\mathrm{NaCl}, 10 \mathrm{~m} M$ imidazole and $0.2 \%(v / v)$ Tween-20. The cells were then disrupted by sonication on ice and the lysate was clarified by centrifugation.

The supernatant was applied onto an $\mathrm{Ni}^{2+}$-charged chelating column equilibrated with lysis buffer without detergent. The protein was eluted with a gradient of imidazole. Fractions containing LMO2:Ldb1-LID were pooled for additional purification using a S75 gel-filtration column (GE Healthcare) with $20 \mathrm{mM}$ Tris $\mathrm{pH} \mathrm{7.4,}$ $300 \mathrm{~m} M \mathrm{NaCl}$ and $1 \mathrm{~m} M$ DTT. Fractions containing LMO2:Ldb1LID were pooled and concentrated to $20 \mathrm{mg} \mathrm{ml}^{-1}$ using Amicon columns.

\subsection{Crystallization and data collection}

All crystallization experiments were performed at $295 \mathrm{~K}$ using the sitting-drop vapour-diffusion method. Initial screening of 768 conditions belonging to various kits from Hampton Research and Emerald BioStructures was carried out using Cartesian Honeybee X8 dispensing robots to pipette $100 \mathrm{nl}$ protein solution and $100 \mathrm{nl}$ precipitant solution into single drops in 96-well Greiner plates (Walter et al., 2005). Crystals appeared after a day and further optimization was performed in 24-well Linbro plates, mixing $2 \mu$ l protein solution with $1 \mu \mathrm{l}$ reservoir solution and equilibrating the drop against $500 \mu \mathrm{l}$ reservoir solution. The best diffracting crystals grew within one week of setup in $1.6 \mathrm{M} \mathrm{NaCl}, 100 \mathrm{~m} M$ citrate $\mathrm{pH} 5$ and $1 \mathrm{~m} M$ DTT (Fig. 2). For data collection, crystals were briefly immersed in $4 M$ sodium malonate $\mathrm{pH} 5.0$ prior to flash-cooling in a nitrogen-gas stream. The $\mathrm{Zn}$ atoms of the LIM zinc-finger domains were used as anomalous scatterers for structure determination using the multiple-wavelength anomalous dispersion (MAD) method. Data sets were collected from a single crystal of LMO2:Ldb1-LID on beamline BM14 at the ESRF, Grenoble, France using a MAR CCD 225 detector with an oscillation of $1^{\circ}$, an exposure time of $60 \mathrm{~s}$ and a crystal-to-detector distance of $242.4 \mathrm{~mm}$. Following a broad X-ray excitation scan of the crystal, data were collected at three wavelengths near the zinc absorption edge (peak $\lambda_{1}=1.28226 \AA$ with $f^{\prime}=-7.5, f^{\prime \prime}=5.4$, inflection $\lambda_{2}=1.28267 \AA$ with $f^{\prime}=-10.2, f^{\prime \prime}=2.9$ and high-energy remote $\lambda_{3}=1.27565 \AA$; Table 1$)$. Indexing, integration and scaling were carried out using $H K L-2000$ (Otwinowski \& Minor, 1996).

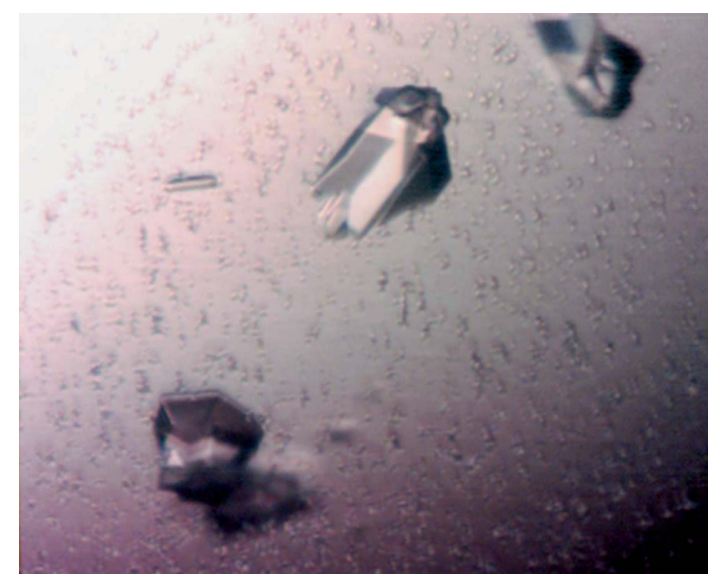

Figure 2

LMO2:Ldb1-LID crystals. Crystal dimensions are approximately $70 \times 50 \times 50 \mu \mathrm{m}$. 


\section{crystallization communications}

\section{Results and discussion}

LMO2:Ldb1-LID was expressed as a soluble $6 \times$ His-tagged protein with a yield of about $5 \mathrm{mg}$ of pure protein per litre of LB culture. Analysis of the data from size-exclusion chromatography indicated that LMO2:Ldb1-LID behaves as a monomer in solution (data not shown). LMO2:Ldb1-LID crystallized in a variety of different conditions, all with a high salt concentration $(>1.5 M)$ and a low $\mathrm{pH}(<6)$. The LMO2:Ldb1-LID crystals (Fig. 2) used for data collection had typical dimensions of $50 \times 50 \times 50 \mu \mathrm{m}$ and appeared to be multiple upon visual inspection. Generally, LMO2:Ldb1-LID crystals diffracted poorly $(<4.0 \AA)$ and analysis of the diffraction data indi- cated the presence of more than one lattice. Consecutive rounds of annealing greatly improved the diffraction quality and resolution limits of the crystals. Following this strategy, it was possible to collect data from a single crystal which diffracted to $2.8 \AA$ resolution and presented only two predominant lattices. A three-wavelength MAD data collection at the $\mathrm{Zn}$ absorption edge was carried out on beamline BM14 using this crystal. Despite the presence of two crystal lattices, the diffraction spots could be indexed and scaled in either crystal orientation (Fig. 3), resulting in two data sets of equal quality (as judged by the data-collection statistics; Table 1). The two data sets could also be scaled together into a single data set belonging to the monoclinic space group $C 2$, with unit-cell parameters $a=179.9$,

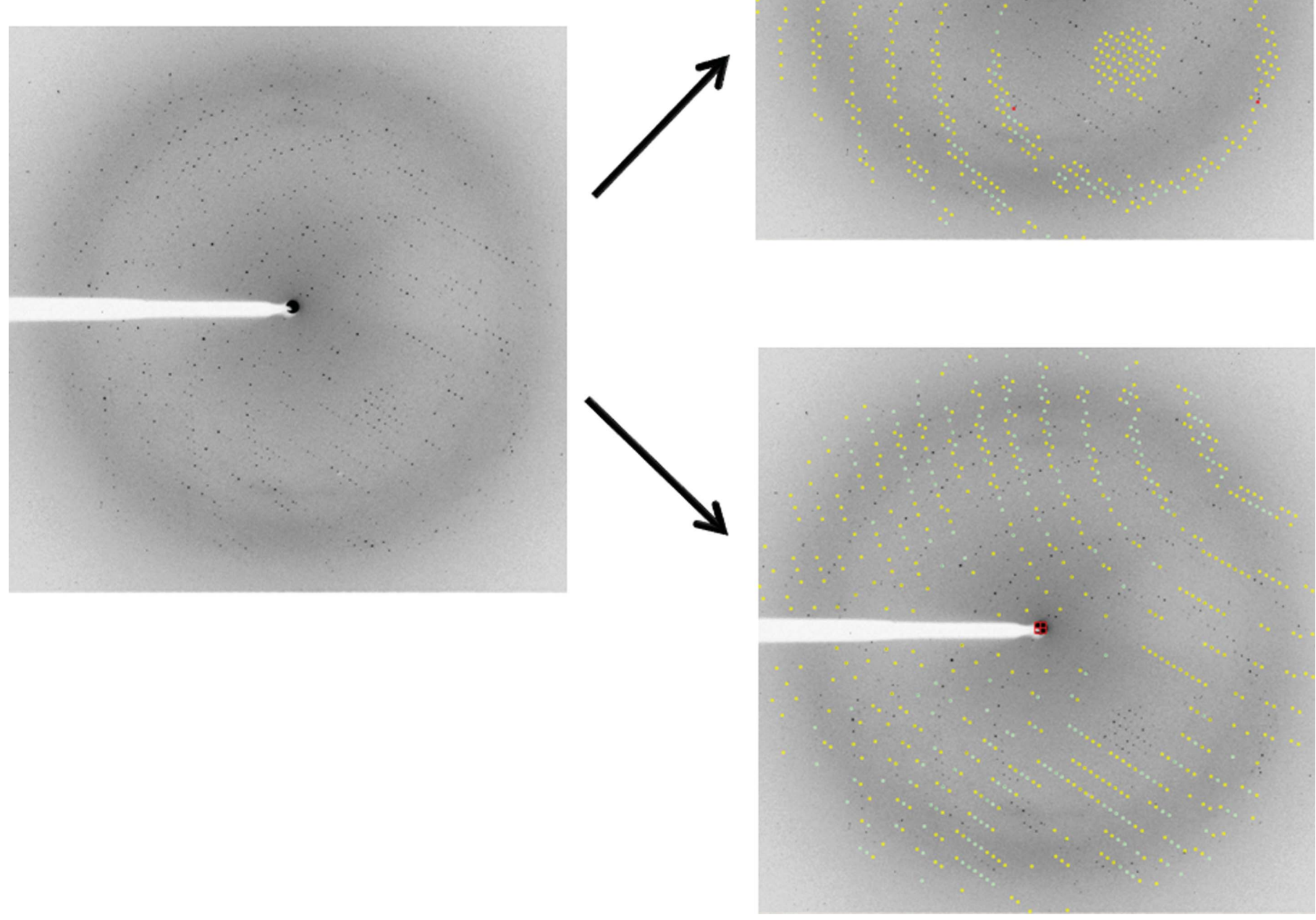

Figure 3

The figure shows two possible indexings of the same diffraction image created by the superimposition of two crystal lattices. Indexing was performed using the program $H K L-2000$. 
Table 1

Data-collection details.

Values in parentheses are for the outer shell.

\begin{tabular}{|c|c|c|c|c|c|c|c|c|c|}
\hline \multirow[b]{2}{*}{ Data } & \multicolumn{3}{|l|}{ Peak } & \multicolumn{3}{|l|}{ Inflection } & \multicolumn{3}{|l|}{ Remote } \\
\hline & Lattice 1 & Lattice 2 & Merged & Lattice 1 & Lattice 2 & Merged & Lattice 1 & Lattice 2 & Merged \\
\hline Space group & $C 2$ & $C 2$ & $C 2$ & $C 2$ & $C 2$ & $C 2$ & $C 2$ & $C 2$ & $C 2$ \\
\hline \multicolumn{10}{|l|}{ Unit-cell parameters } \\
\hline$a(\AA)$ & 179.9 & 179.9 & 180.3 & 180.3 & 180.5 & 180.3 & 180.2 & 180.3 & 180.3 \\
\hline$b(\AA)$ & 55.5 & 55.5 & 55.8 & 55.8 & 55.7 & 55.8 & 55.7 & 55.6 & 55.6 \\
\hline$c(\AA)$ & 114.7 & 114.8 & 114.8 & 114.9 & 114.9 & 114.9 & 114.9 & 114.9 & 114.9 \\
\hline$\beta\left(^{\circ}\right)$ & 90.1 & 90.1 & 90.1 & 90.1 & 90.1 & 90.1 & 90.1 & 90.1 & 90.1 \\
\hline Wavelength $(\AA)$ & 1.28226 & 1.28226 & 1.28226 & 1.28267 & 1.28267 & 1.28267 & 1.27565 & 1.27565 & 1.27565 \\
\hline Resolution (A) & $50-2.8$ & $50-2.9$ & $50-2.9$ & $50-3.0$ & $50-3.1$ & $50-3.1$ & $50-2.9$ & $50-3.0$ & $50-2.9$ \\
\hline Completeness (\%) & $96.9(76.7)$ & $99.7(97.9)$ & $100(100)$ & $98.1(99.1)$ & $96.4(100)$ & $100(100)$ & $97.5(88.9)$ & $95.3(100)$ & $100(100)$ \\
\hline Redundancy & $8.9(7.5)$ & $7.5(6.8)$ & $18.3(16.2)$ & $3.7(3.8)$ & $3.6(3.7)$ & $7.3(7.5)$ & $3.8(3.4)$ & $3.7(3.9)$ & $7.4(6.3)$ \\
\hline$I / \sigma(I)$ & $41.5(5.0)$ & $27.8(2.4)$ & $31.5(4.7)$ & $11.7(1.3)$ & $9.5(1.3)$ & $16.9(2.4)$ & $12.1(1.3)$ & $8.2(1.2)$ & $15.4(1.6)$ \\
\hline$R_{\text {merge }}(\%)$ & $15.2(87.2)$ & $20.8(94.7)$ & $18.7(91.5)$ & 14.3 (91.6) & $19.9(93.3)$ & $17.8(88.6)$ & $14.2(82.7)$ & $20.2(91.7)$ & $19.3(98.3)$ \\
\hline
\end{tabular}

$b=51.5, c=114.7 \AA, \beta=90.1^{\circ}$ (Table 1 ). Despite a $\beta$-angle value very close to $90^{\circ}$, it was not possible to scale the data in orthorhombic space groups. Matthews coefficient analysis predicted five LMO2:Ldb1-LID molecules per asymmetric unit of the crystal, with a $V_{\mathrm{M}}$ value of $2.4 \AA^{3} \mathrm{Da}^{-1}$ and a solvent content of $49 \%$ (Matthews, 1968).

Molecular-replacement algorithms (MOLREP and Phaser; Vagin \& Teplyakov, 1997; McCoy, 2007) were unable to solve the structure of LMO2:Ldb1-LID despite the availability of a $50 \%$ identical model (LMO4:Ldb1-LID; PDB code 1rut; Deane et al., 2004). As well as both LIM domains together, the individual LIM domains of LMO4 were unsuccessfully used as search models, suggesting a difference in the configuration of the LMO2 and LMO4 proteins. Despite the strong anomalous signal from the naturally occurring $\mathrm{Zn}$ atoms in LMO2:Ldb1-LID (four zincs per LMO2:Ldb1-LID, 20 zincs per asymmetric unit) as judged by the anomalous signal of $6.9-8 \AA$ observed in SHELXC (Pape \& Schneider, 2004), it was initially not possible to find the positions of the $\mathrm{Zn}$ atoms when using either of the two individual data sets. Only when the two data sets were scaled and merged together (Table 1) was it possible to solve the anomalous scatterer substructure by determining and refining the position of the $20 \mathrm{Zn}$ atoms per asymmetric unit with an overall figure of merit of 0.7 . The serendipitous presence of the two lattices aided structure solution by providing us with the possibility of collecting twice the number of reflections from the outset, minimizing the radiation damage and improving the recorded anomalous signal. Zinc-site determination, phasing, density modification and initial model building were performed using PHENIX AutoSol and AutoBuild (Adams et al., 2002). More recently, crystals of LMO2:Ldb1-LID displaying a different morphology (plates) and belonging to space $P 2_{1}$ were obtained that contained one molecule in the asymmetric unit and diffracted to a resolution of $2.4 \AA$. Structure refinement and analysis of the two crystal forms is under way.

We are grateful to Martin Walsh, Hassan Belrhali and Mario Bumann at the BM14 UK MAD beamline (ESRF, Grenoble, France) for assistance with data collection. This work was supported by Leukaemia and Lymphoma Research. EJM is a Royal Society University Research Fellow and CP is supported by the Medical Research Council.

\section{References}

Adams, P. D., Grosse-Kunstleve, R. W., Hung, L.-W., Ioerger, T. R., McCoy, A. J., Moriarty, N. W., Read, R. J., Sacchettini, J. C., Sauter, N. K. \& Terwilliger, T. C. (2002). Acta Cryst. D58, 1948-1954.
Appert, A., Nam, C.-H., Lobato, N., Priego, E., Miguel, R. N., Blundell, T., Drynan, L., Sewell, H., Tanaka, T. \& Rabbitts, T. (2009). Cancer Res. 69, 4784-4790.

Bach, I. (2000). Mech. Dev. 91, 5-17.

Boehm, T., Foroni, L., Kaneko, Y., Perutz, M. F. \& Rabbitts, T. H. (1991). Proc. Natl Acad. Sci. USA, 88, 4367-4371.

Deane, J. E., Mackay, J. P., Kwan, A. H., Sum, E. Y., Visvader, J. E. \& Matthews, J. M. (2003). EMBO J. 22, 2224-2233.

Deane, J. E., Ryan, D. P., Sunde, M., Maher, M. J., Guss, J. M., Visvader, J. E. \& Matthews, J. M. (2004). EMBO J. 23, 3589-3598.

Deane, J. E., Sum, E., Mackay, J. P., Lindeman, G. J., Visvader, J. E. \& Matthews, J. M. (2001). Protein Eng. 14, 493-499.

Ferrando, A. A., Herblot, S., Palomero, T., Hansen, M., Hoang, T., Fox, E. A. \& Look, A. T. (2004). Blood, 103, 1909-1911.

Grutz, G. G., Bucher, K., Lavenir, I., Larson, T., Larson, R. \& Rabbitts, T. H. (1998). EMBO J. 17, 4594-4605.

Herblot, S., Steff, A. M., Hugo, P., Aplan, P. D. \& Hoang, T. (2000). Nature Immunol. 1, 138-144.

Jurata, L. W. \& Gill, G. N. (1997). Mol. Cell. Biol. 17, 5688-5698.

Kadrmas, J. L. \& Beckerle, M. C. (2004). Nature Rev. Mol. Cell Biol. 5, 920-931.

Lecuyer, E., Lariviere, S., Sincennes, M. C., Haman, A., Lahlil, R., Todorova, M., Tremblay, M., Wilkes, B. C. \& Hoang, T. (2007). J. Biol. Chem. 282, 33649-33658.

Ma, S., Guan, X. Y., Beh, P. S., Wong, K. Y., Chan, Y. P., Yuen, H. F., Vielkind, J. \& Chan, K. W. (2007). J. Pathol. 211, 278-285.

Matthews, B. W. (1968). J. Mol. Biol. 33, 491-497.

McCoy, A. J. (2007). Acta Cryst. D63, 32-41.

Nam, C.-H., Lobato, M. N., Appert, A., Drynan, L. F., Tanaka, T. \& Rabbitts, T. H. (2008). Oncogene, 27, 4962-4968.

Natkunam, Y., Zhao, S., Mason, D. Y., Chen, J., Taidi, B., Jones, M., Hammer, A. S., Hamilton Dutoit, S., Lossos, I. S. \& Levy, R. (2007). Blood, 109, 16361642.

Ono, Y., Fukuhara, N. \& Yoshie, O. (1998). Mol. Cell. Biol. 18, 69396950.

Otwinowski, Z. \& Minor, W. (1996). Methods Enzymol. 276, 307326.

Pape, T. \& Schneider, T. R. (2004). J. Appl. Cryst. 37, 843-844.

Ryan, D. P., Sunde, M., Kwan, A. H., Marianayagam, N. J., Nancarrow, A. L., Vanden Hoven, R. N., Thompson, L. S., Baca, M., Mackay, J. P., Visvader, J. E. \& Matthews, J. M. (2006). J. Mol. Biol. 359, 66-75.

Schlaeger, T. M., Schuh, A., Flitter, S., Fisher, A., Mikkola, H., Orkin, S. H., Vyas, P. \& Porcher, C. (2004). Mol. Cell. Biol. 24, 7491-7502.

Vagin, A. \& Teplyakov, A. (1997). J. Appl. Cryst. 30, 1022-1025.

Wadman, I. A., Osada, H., Grutz, G. G., Agulnick, A. D., Westphal, H., Forster, A. \& Rabbitts, T. H. (1997). EMBO J. 16, 3145-3157.

Walter, T. S. et al. (2005). Acta Cryst. D61, 651-657.

Warren, A. J., Colledge, W. H., Carlton, M. B., Evans, M. J., Smith, A. J. \& Rabbitts, T. H. (1994). Cell, 78, 45-57.

Yamada, Y., Pannell, R., Forster, A. \& Rabbitts, T. H. (2000). Proc. Natl Acad. Sci. USA, 97, 320-324.

Yamada, Y., Warren, A. J., Dobson, C., Forster, A., Pannell, R. \& Rabbitts, T. H. (1998). Proc. Natl Acad. Sci. USA, 95, 3890-3895.

Zheng, Q. \& Zhao, Y. (2007). Biol. Cell, 99, 489-502. 